\title{
Tracing the evolution of amniote chromosomes
}

\author{
Janine E. Deakin • Tariq Ezaz
}

Received: 20 December 2013 / Revised: 3 March 2014 / Accepted: 4 March 2014 /Published online: 25 March 2014

(C) The Author(s) 2014. This article is published with open access at Springerlink.com

\begin{abstract}
A great deal of diversity in chromosome number and arrangement is observed across the amniote phylogeny. Understanding how this diversity is generated is important for determining the role of chromosomal rearrangements in generating phenotypic variation and speciation. Gaining this understanding is achieved by reconstructing the ancestral genome arrangement based on comparisons of genome organization of extant species. Ancestral karyotypes for several amniote lineages have been reconstructed, mainly from cross-species chromosome painting data. The availability of anchored whole genome sequences for amniote species has increased the evolutionary depth and confidence of ancestral reconstructions from those made solely from chromosome painting data. Nonetheless, there are still several key lineages where the appropriate data required for ancestral reconstructions is lacking. This review highlights the progress that has been made towards understanding the chromosomal changes that have occurred during amniote evolution and the reconstruction of ancestral karyotypes.
\end{abstract}

Keywords Comparative genomics · Chromosome painting · Ancestral karyotype $\cdot$ Reptile $\cdot$ Bird $\cdot$ Mammal

\section{Introduction}

Chromosomes, the basic units into which DNA is packaged in a nucleus, have undergone changes in gene content and organization throughout evolution. The great diversity of chromosome numbers between different amniote species and even the contrasting division of macro and microchromosomes in most

J. E. Deakin $(\bowtie) \cdot$ T. Ezaz

Institute for Applied Ecology, University of Canberra,

Canberra 2601, Australia

e-mail: Janine.Deakin@canberra.edu.au birds and non-avian reptiles presents an opportunity to study chromosome evolution to determine the timing and types of events that shaped the chromosomes of extant amniote species. This involves comparing chromosomes of different species to reconstruct the most likely chromosome arrangement in a common ancestor. Tracing such events can provided great insight into the evolutionary process and even the role chromosomal rearrangements play in phenotypic evolution and speciation.

Reconstruction of ancestral karyotypes at various positions along the amniote (reptiles, birds and mammals) phylogenetic tree has been made possible by the large number of crossspecies chromosome painting and gene mapping studies that have been carried out over the last 20 years, and more recently from the availability of sequenced and anchored genomes. Reconstructions based on cross-species chromosome painting data provide the most basic ancestral plan, only permitting the arrangement of relatively large-scale evolutionary events to be traced. The limits of detection of chromosome painting also govern the evolutionary depth to which the reconstruction can be applied. For instance, chromosome paints generated from eutherian species (e.g. humans) fail to detect homology with marsupial or monotreme chromosomes (Graphodatsky et al. 2012) and therefore, ancestral karyotype reconstructions based solely on eutherian chromosome painting data are restricted to the eutherian lineage. Interestingly, a greater evolutionary depth is possible in the reptilian lineage, with chromosome probes derived from chicken able to detect homology to crocodile, turtle and lizard species (Kasai et al. 2012; Pokorna et al. 2012; Pokorna et al. 2011), which last shared a common ancestor over 200 million years ago (MYA). Combining chromosome painting data with gene mapping or whole genome data permits reconstructions over greater evolutionary time, enabling the evolutionary events that have occurred across amniotes to be determined. However, at this stage, there are only a limited number of species for which the appropriate 
detailed data is available. In addition, the species that have been mapped or sequenced are not necessarily the best representative species for a particular lineage, which could complicate the reconstruction process. Basically, there is no single approach that is able to provide all the answers but it is a matter of aptly using the available data.

Here, we review the advancements that have been made in this field using molecular cytogenetics and comparative genomics analysis, highlighting the data missing from key species and suggest approaches that can be taken to rapidly bridge these knowledge gaps.

\section{Amniote chromosome numbers}

Amniotes, which include birds, non-avian reptiles (herein referred to as reptiles) and mammals, last shared a common ancestor approximately 310 MYA. The chromosomes of each of these three major amniote lineages are strikingly different (Fig. 1), suggesting that their genomes have been subject to considerable rearrangement since last sharing a common ancestor.

Karyotypes of birds and most reptiles consist of up to ten pairs of macrochromosomes and a varying number of microchromosomes (Table 1). Birds have a particularly large number of microchromosomes. Reptiles are considered to be a karyologically heterogeneous group displaying high diversity in chromosome numbers and morphologies between and among groups (Olmo 2008). Among reptiles, crocodilians (Cohen and Gans 1970; Olmo 2008) and turtles (Olmo 2008; Olmo and Signorino 2005; Valenzuela and Adams 2011) have the most conserved karyotypes. In contrast, squamates reptiles (snakes and lizards, including legless lizards) have a high level of karyotypic variability in both morphologies and numbers observed. Snakes have relatively conserved chromosome numbers, which typically include eight pairs of macrochromosomes and ten pairs of microchromosomes. However, karyotypes have undergone frequent rearrangements including fission, fusion and repeat accumulation (Mengden and Stock 1980; O’Meally et al. 2010). Lizards (including legless lizards) seem to show the highest karyotypic variability among reptiles.

Mammalian karyotypes vary greatly between the prototherians (monotremes), metatherians (marsupials) and eutherians (placentals). Monotremes have karyotypes with high diploid numbers (Table 1). At first glance, it may appear that monotreme karyotypes are similar to those of reptilian species with eight large and many small chromosomes (Matthey 1949). However, the chromosome size range is much more continuous than that typically observed in reptiles and birds, and the small chromosomes are much larger than tiny microchromosomes (Van Brink 1959). In contrast, marsupial karyotypes mostly consist of several large chromosomes. Eutherian mammals show the most karyotypic diversity (Table 1) (O'Brien et al. 2006) and particular eutherians lineages, such as the rodents, gibbons, and canines have experienced extensive chromosome rearrangement.

Tracing the evolutionary history of amniote chromosomes will help us to understand the events that have led to the extraordinary diversity in karyotype morphology and chromosome number observed. This involves establishing the chromosome homology between representative species and reconstructing key ancestral karyotypes.

\section{The ancestral avian karyotype}

Avian karyotypes are divided into macro- and microchromosomes, with the macrochromosomes being relatively gene poor compared to the gene-rich microchromosomes (Burt 2002). In chicken (Gallus gallus), the most well-studied avian genome, the macrochromosomes make up approximately $70 \%$ of the genome (Kasai et al. 2012). Chromosome homology between different avian orders has revealed a striking level of conservation of macrochromosomes between divergent taxa (Griffin et al. 2007). Cross-species chromosome painting, typically using probes generated from chicken chromosomes 1 to 9 and often the large $\mathrm{Z}$ chromosome, have shown that, in many cases, whole chromosomes have remained intact (de Oliveira et al. 2008; Derjusheva et al. 2004; Nanda et al. 2011; Nishida et al. 2008). This remarkable conservation is not restricted to the avian lineage but is even observed in outgroup species such as crocodiles and turtles (Kasai et al. 2012), which last shared a common ancestor with birds 230 MYA. The homology between chicken chromosomes and those of representatives of various avian orders is shown in Fig. 2. The high level of conservation has made it relatively easy to predict the ancestral avian karyotype (Griffin et al. 2007), even despite the uncertainty surrounding avian phylogeny and their relationship to turtles and crocodiles (Kasai et al. 2012).

Despite the infrequent occurrence of interchromosomal rearrangements detected by chromosome painting, comparisons of gene order from gene maps or sequenced and anchored genomes has demonstrated that there are intrachromosomal rearrangements (Skinner and Griffin 2012; Volker et al. 2010). This is exemplified by the comparison of the genome assemblies for chicken, zebra finch (Taeniopygia guttata) and turkey (Meleagris gallopavo), where inversions are evident and particular regions of the genome appear to be prone to rearrangement (Skinner and Griffin 2012). The Z chromosome is one chromosome that has been particularly susceptible to intrachromosomal rearrangements (Griffin et al. 2007). It is unclear why bird genomes are more prone to intrachromosomal than interchromosomal rearrangement but Skinner and Griffin (2012) propose that there may be an advantage for birds maintaining the association of certain 
Fig. 1 Amniote phylogeny showing haploid karyotypes for representative species. The range of haploid chromosomes numbers for each lineage are indicated on the branches (Christidis 1990; Hayman 1990; O’Brien et al. 2006; Olmo and Signorino 2005; Valenzuela and Adams 2011).

Microchromosomes are indicated in dark grey. The sex chromosomes present in the homogametic sex are shown for representative species and alternatives present in each lineage are indicated. TSD temperature sex determination

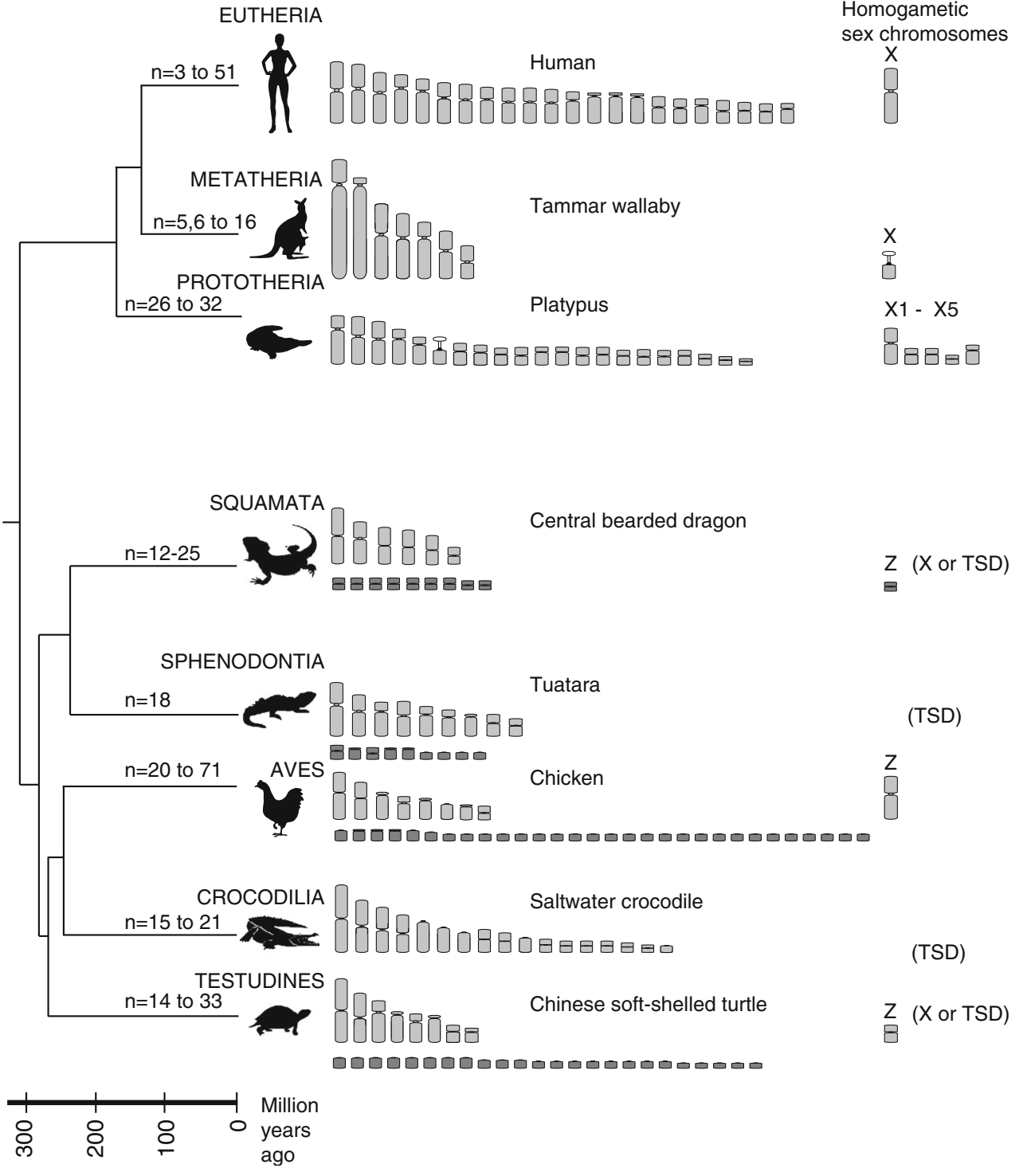

Table 1 The range of diploid numbers in amniotes (Christidis 1990; Hayman 1990; O'Brien et al. 2006; Olmo and Signorino 2005; Valenzuela and Adams 2011)

\begin{tabular}{lllll}
\hline & \multicolumn{2}{l}{ Diploid chromosome numbers } & \multirow{2}{*}{ Microchromosomes } \\
\cline { 2 - 3 } & Smallest & Largest & Common $^{\mathrm{a}}$ & \\
\hline Birds & 40 & $136-142$ & 80 & Yes \\
Crocodiles & 30 & 42 & & No \\
Turtles & 28 & 66 & & Yes \\
Snakes & 30 & 42 & 36 & Yes \\
Lizards & 24 & 46 & & Yes $^{\mathrm{b}}$ \\
Legless Lizards & 30 & 50 & & Yes \\
Monotremes & 52 & 64 & & No \\
Marsupials & 10 & 32 & 14 and 22 & No \\
Eutherians & 6 & 102 & & No \\
\hline
\end{tabular}

${ }^{a}$ Only given for groups were there is a common diploid number

${ }^{\mathrm{b}}$ Absent from some species syntenic blocks in interphase nuclei. Nonetheless, intrachromosomal rearrangements are capable of bringing about phenotypic change and therefore, may play an important role in speciation in the avian lineage. For example, an inversion on chromosome 2 in the white-throated sparrow (Zonotrichia albicollis) is responsible for behavioural differences in the population, providing an example of phenotypic variation generated by an intrachromosomal rearrangement and may even be an example of speciation in action (Thomas et al. 2008). Avian genome evolution has been thoroughly reviewed in recent years and we refer the reader to these reviews for further details (Ellegren 2010; Griffin et al. 2007).

Avian microchromosomes

Evolution of bird macrochromosomes is now relatively well understood. However, our understanding of microchromosome evolution is lagging. Microchromosomes 
Fig. 2 Homology identified in representative species across different avian lineages and outgroups (crocodile and turtle) using cross-species chromosome painting with probes derived from chicken chromosomes 1 to 9 (depicted by different colours). The predicted ancestral avian karyotype is indicated (Griffin et al. 2007). Chromosome homology was sourced from: T.s. elegans and C. niloticus (Kasai et al. 2012); D. novaehollandiae (Shetty et al. 1999); A. sponsa, F. atra and S. atricapilla (Nanda et al. 2011); C. livia (Derjusheva et al. 2004); P. perspicillata (de Oliveira et al. 2008); F. columbarius (Nishida et al. 2008); A. roseicollis (Nanda et al. 2007). $m$ microchromosome

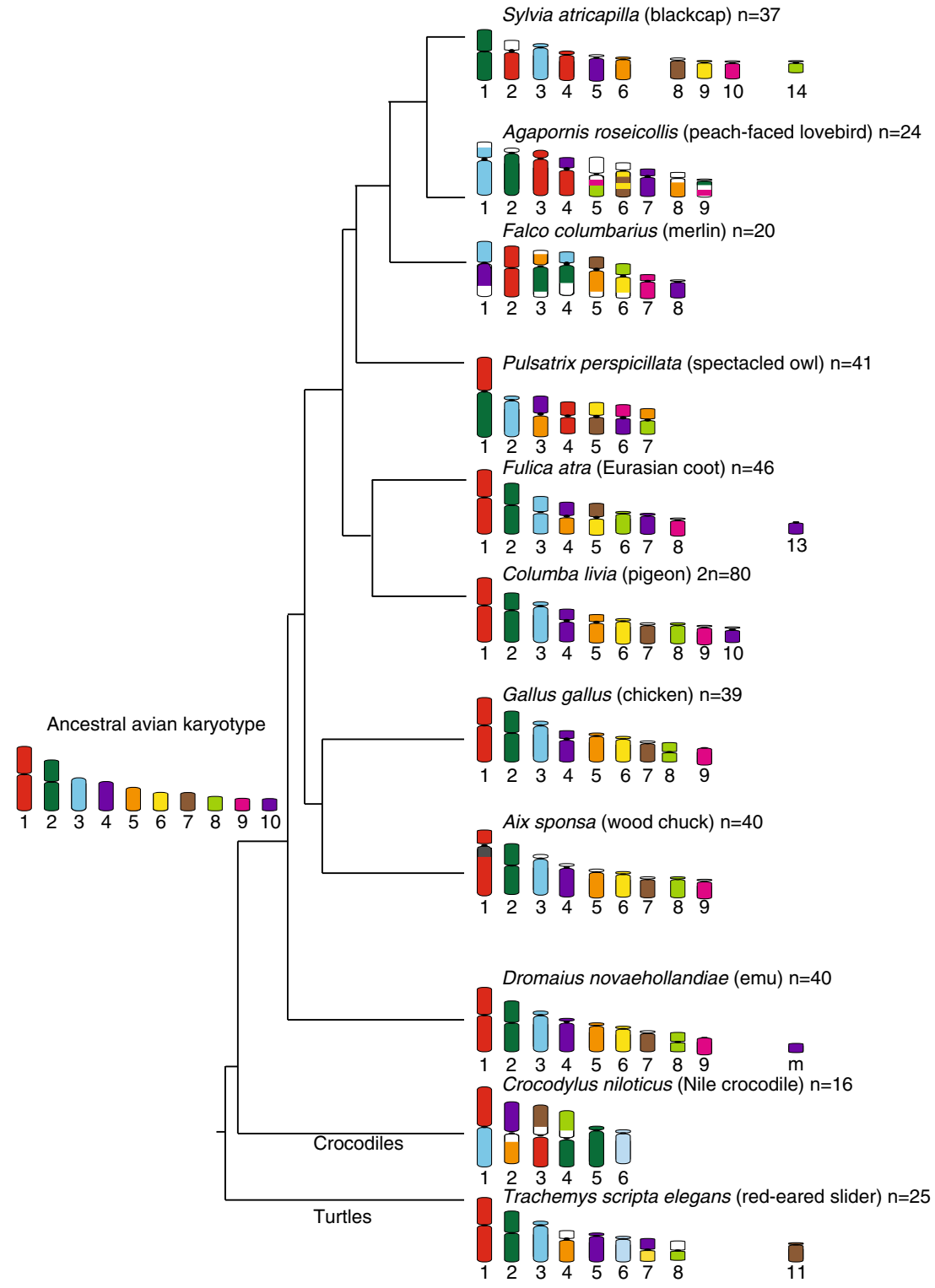

The limited number of studies using chicken microchromosome paints on other bird species has revealed that, in most cases, single microchromosomes in chicken are homologous to single microchromosomes in other species or probes derived from pools of chicken microchromosomes hybridise to the same number of microchromosomes in other species, such as goose (Griffin et al. 1999), pheasant (Phasanius colchicus) (Grutzner et al. 2001), turkey (Griffin et al. 2008), pigeon (Columba livia), chaffinch (Fringilla coelebs) and redwing (Turdus iliacus) (Derjusheva et al. 2004). This implies that synteny is conserved for microchromosomes, which is supported by mapping of chicken bacterial artificial chromosome (BAC) clones to duck (Anas platyrhynchos) (Fillon et al. 2007; Skinner et al. 2009) and quail (Coturnix japonica) (Kayang et al. 2006) 
microchromosomes, where pairs of BACs marking opposite ends of chicken microchromosomes mapped to single microchromosomes in the duck or quail.

Exceptions to this level of conservation can be found in species with atypical avian karyotypes, such as the stone curlew (Burhinus oedicnemus) (Nie et al. 2009), a member of a phylogenetically young group of birds with a low diploid number of $2 n=42$ or the Harpy eagle (Harpia harpyja) with a $2 n=58$ karyotype (de Oliveira et al. 2005). Stone curlew medium-size chromosomes share homology mostly with chicken microchromosomes, indicating that fusions contributed significantly to shaping the curlew karyotype (Nie et al. 2009). This is also supported by the hybridization of stone curlew chromosome paints onto chromosomes from species representing five different avian orders (Hansmann et al. 2009). In the Harpy eagle, Japanese mountain hawk-eagle (Nisaetus nipalensis orientalis) and three falcon species (Falco tinnunculus, Falco peregrinus, Falco columbarius), chromosome painting revealed tandem fusions between microchromosomes, as well as between microchromosomes and macrochromosomes (de Oliveira et al. 2005; Nishida et al. 2013; Nishida et al. 2008). This is also likely to be the case in the parrot Agapornis roseicollis, where there is some evidence of this from mapping of a chicken microchromosome probe to a macrochromosome (Nanda et al. 2007).

These interesting tiny, gene-rich chromosomes need to be the focus of future studies in order to more fully understand the evolution of avian genomes. A comparison of chicken and turkey intronic and cDNA sequence has indicated that microchromosomes have a higher rate of sequence evolution than the larger chromosomes, most likely influenced by the higher incidence of $\mathrm{CpG}$ sites on microchromosomes (Axelsson et al. 2005). It would be interesting to expand this type of analysis to many more species, particularly to species where fusions of ancestral microchromosomes have occurred. In order to do this, it is essential to have sequence anchored to chromosomes. Of the 11 bird genomes published to date, only a third of these have been at least partially anchored to chromosomes (Table 2). Although the growing list of bird genomes being sequenced using next generation technology will be valuable resources, their usefulness for understanding avian and amniote genome evolution will be limited without the input of additional information (Lewin et al. 2009).

\section{Sex chromosome evolution}

All birds have a ZZ male/ZW female sex chromosome system, with the $\mathrm{Z}$ chromosome conserved across Aves, from the Palaeognathae (Struthioniformes and Tinamiformes), such as the emu (Nishida-Umehara et al. 2007; Shetty et al. 1999), to the Neognathae, including those with more atypical avian karyotypes, such as falcons (Nishida et al. 2008). This indicates that avian sex chromosomes arose prior to the divergence of the Palaeognathae and Neognathae lineages. The $\mathrm{W}$ chromosome differs in size between different avian species, being almost identical to the $\mathrm{Z}$ in size in Palaeognathae, to being smaller and heterochromatic in many Neognathae (reviewed in Stiglec et al. 2007). In contrast to the more highly differentiated ZW pairs of Neognathae species, Palaeognathae $\mathrm{Z}$ and $\mathrm{W}$ chromosomes recombine over a large portion of the chromosome (Pigozzi and Solari 1997). However, the apparent lack of differentiation over the last 100 million years is not solely due to this recombination but may be a consequence of the absence of a dosage compensation mechanism (required to equalize Z-gene expression between males and females) in these species. In fact, the lack of such a mechanism may have constrained sex chromosome differentiation so that two copies of most genes, particularly those that are dosage sensitive, are maintained on the $\mathrm{W}$ chromosome (Adolfsson and Ellegren 2013).

Sequencing information of the $\mathrm{Z}$ and $\mathrm{W}$ chromosomes of different avian species will provide a more in-depth understanding of the evolution of these chromosomes. However, the challenge is in obtaining the highly repetitive $\mathrm{W}$ sequence. The assembly of chicken $\mathrm{W}$ in the published chicken genome covers only a tiny fraction $(0.5 \%)$ of the entire chromosome (International Chicken Genome Sequencing C 2004). About $7 \%$ of the ZW genome has not been anchored to a chromosome and therefore is a source for potential W-specific sequence. By sequencing a male genome using the Illumina sequencing platform and aligning reads to the assembled ZW genome, more $\mathrm{W}$-specific sequences have been identified (Chen et al. 2012). Hopefully, similar bioinformatic approaches and the continued development of sequencing technology capable of sequencing through repetitive regions will lead to a thorough understanding of bird sex chromosome evolution.

\section{Towards understanding the evolution of reptile genomes}

Reptiles occupy a key position in vertebrate phylogeny by sharing the common ancestor to birds and mammals (Fig. 1). Therefore, they are likely to play a critical role by providing fundamental and basic information to better understand genome organization and evolution in birds and mammals. The first step in determining how the genomes of this karyotypically diverse group have evolved is to establish the level of conservation between different reptilian species, as well as more broadly with birds and mammals. Progress in this area has been slow compared to birds and mammals, but is rapidly gaining pace in this post-genomics era.

In comparison to birds and mammals, only a limited number of cross-species chromosome painting studies have been carried out to detect the level of conservation among particular groups of reptiles (Giovannotti et al. 2009; Pokorna et al. 
Table 2 Published bird genome assemblies

Percentage of genome assembly anchored to chromosomes

${ }^{\mathrm{b}}$ Anchored to chromosomes or linkage groups

\begin{tabular}{|c|c|c|c|}
\hline Species & Common name & $\begin{array}{l}\text { Percent } \\
\text { anchored }^{\mathrm{a}}\end{array}$ & Reference \\
\hline Gallus gallus & Chicken (red jungle fowl) & 89 & $\begin{array}{l}\text { International Chicken Genome } \\
\text { Sequencing C } 2004\end{array}$ \\
\hline Taeniopygia guttata & Zebra finch & $83^{\mathrm{b}}$ & Warren et al. 2010 \\
\hline Meleagris gallopavo & Wild turkey & 83 & Dalloul et al. 2010 \\
\hline Ficedula albicollis & Collared flycatcher & 73 & Ellegren et al. 2012 \\
\hline Anas platyrhynchos domestica & Pekin duck & 26 & Huang et al. 2013 \\
\hline Amazona vittata & Puerto Rican parrot & - & Oleksyk et al. 2012 \\
\hline Ara macao & Scarlet macaw & - & Seabury et al. 2013 \\
\hline Columba livia & Domestic rock pigeon & - & Shapiro et al. 2013 \\
\hline Falco cherrug & Saker falcon & - & Zhan et al. 2013 \\
\hline Falco peregrinus & Peregrine falcon & - & Zhan et al. 2013 \\
\hline Geospiza magnirostris & Large ground finch & - & Rands et al. 2013 \\
\hline
\end{tabular}

2012; Trifonov et al. 2011). Nonetheless, these studies also discovered a gross level of conservation between avian and squamate genomes, suggesting retention of large homologous synteny blocks (HSBs) throughout the evolutionary history of reptiles (Pokorna et al. 2012). Cross-species chromosome painting has also been used to decipher inter-specific genome evolution in skinks (Scincidae), which detected strong conservation of chromosomes between five species with variable chromosome numbers, suggesting a monophyletic origin of the family Scincidae (Giovannotti et al. 2009). Similarly, cross-species chromosome painting in seven species of geckos, not only detected highly conserved karyotypes but also detected species specific rearrangements in the common house gecko (Hemidactylus frenatus) (Trifonov et al. 2011).

Reptiles in the era of comparative genomics

Compared to other vertebrates, reptile genomes have had a late start in the area of comparative genomics but are rapidly catching up. Although whole genome sequences are available (Table 3) or in progress for an increasing list of reptilian species, none of these genomes, except that of the green anole (Anolis carolinensis), have been anchored to chromosomes, and even then only about $60 \%$ of the sequence has been anchored (Alfoldi et al. 2011). Conversely, the genomes of most reptiles for which there are molecular cytogenetic maps (Table 4) are yet to undergo whole genome sequencing. These chromosome maps have provided significant insight into genome organization and evolution in amniotes, identifying an unprecedented level of conservation of amniote genomes. For example, gene mapping data in six reptiles revealed a high level of karyotypic conservation between birds and reptiles, implying retention, to a large degree, of the ancestral karyotype (Uno et al. 2012).

Recently, Young et al. (2013) developed a cytogenetic map in an agamid lizard, the central bearded dragon (Pogona vitticeps) where every single chromosome, including the microchromosomes was anchored with at least one BAC clone/gene. This BAC anchored cytogenetic map provided a unique opportunity to perform high-resolution comparative genomic studies with the capacity to identify fine scale rearrangements. For example, this study revealed an intrachromosomal rearrangement on the long arm of chromosome 1 of $P$. vitticeps $1 \mathrm{q}$ and identified regions orthologous to the Chinese soft-shelled turtle, Pelodiscus sinensis ZW and lizard A. carolinensis XY sex chromosomes (Young et al. 2013).

\section{Reptile microchromosomes}

One of the characteristic features of most reptilian karyotypes is the presence of a variable number of microchromosomes. Compared to avian microchromosomes, only little is known about the genomics of those from reptile species. Classical staining studies in many reptiles provided evidence of the presence of GC-rich sequences on microchromsomes, which are therefore likely to be gene-rich like their avian counterparts. However, staining studies in tuatara showed evidence of AT-rich sequences on microchromosomes (O'Meally et al. 2009). Hence, microchromosomes may have evolved independently multiple times in different lineages.

Analysis of reptilian microchromosomes at the sequence level, and subsequent comparisons with avian microchromosomes, thus presents an excellent opportunity to discover novel insight into their evolution. This has been performed for the green anole, where sequence has been assigned to six microchromosomes, and displays conserved synteny exclusively with chicken microchromosomes. Unlike the higher $\mathrm{GC}$ content of avian microchromosomes, there was no GC content difference between macro- and microchromosomes in the green anole (Alfoldi et al. 2011). Partial sequence, gene, and repeat content has also been 
Table 3 Published reptile genomes

${ }^{\text {a }}$ Percentage of genome assembly anchored to chromosomes

\begin{tabular}{llll}
\hline Species & Common name & Percent anchored $^{\mathrm{a}}$ & Reference $^{\text {Anolis carolinensis }}$ \\
Pelodiscus sinensis & Green anole lizard & 60 & Alfoldi et al. 2011 \\
Chelonia mydas & Soft-shelled turtle & - & Wang et al. 2013 \\
Chrysemyspicta bellii & Green sea turtle & - & Wang et al. 2013 \\
Python molurus bivittatus & Western painted turtle & - & Shaffer et al. 2013 \\
Ophiophagus hannah & Burmese python & - & Castoe et al. 2013 \\
\hline
\end{tabular}

determined for BAC clones mapping to the $\mathrm{W}$ chromosome (a microchromosome) and one mapping to an autosomal microchromosome in P. vitticeps (Ezaz et al. 2013). Such analysis incorporating more species is being made easier due to the advancement of cytogenetic techniques (such as manual microdissection), genome amplification and subsequent sequencing (e.g. Illumina) to generate quality sequence information for comparative studies. Sequencing chromosomes from DNA amplified from a single microchromosome has been found to produce higher quality sequence data compared to that from flow sorted microchromosomes (Ezaz, unpublished). This combined approach of microdissection and sequencing will identify gross homologies of microchromosomes between species and groups, as well as sequence composition, will help us to discover the occurrence of fine scale rearrangements and overall gene composition of microchromosomes.

\section{Sex chromosomes in reptiles}

Squamate reptiles epitomize an extremely high level of diversity regarding sex chromosome morphologies, ranging from cryptic (homomorphic), requiring the high-resolution cytogenetic technique of comparative genome hybridization (CGH) to identify them (Ezaz et al. 2005), to highly differentiated sex chromosomes, with these extremes often observed even between closely related species (Ezaz et al. 2009a; Ezaz et al. 2009b; Olmo and Signorino 2005). Yet, only scant genomic information on sex chromosomes and their evolution is available in reptiles. Both male (XY) and female (ZW) heterogametic systems exist in reptiles including multiple sex chromosomes (Ezaz et al. 2009a; Ezaz et al. 2009b; Olmo and Signorino 2005).

Among reptiles, all snakes have ZW sex chromosomes and comparative gene mapping shows that ZW sex chromosomes are conserved across snake lineages (Matsubara et al. 2012; Matsubara et al. 2006). Lizards and turtles, however, have both $\mathrm{XY}$ and ZW sex chromosome systems and comparative sex chromosome gene mapping revealed that all lizard and turtle sex chromosomes are non-homologous, implying independent origins of sex chromosomes in these lineages (Ezaz et al. 2009a; Kawagoshi et al. 2012; Kawai et al. 2009; Matsubara et al. 2012; Matsubara et al. 2006; Pokorna et al. 2011).

Compared to whole genome mapping, sex chromosomes (both XY and ZW) of reptiles have been relatively well explored, in particular, comparisons with the chicken $Z$ chromosome. Chromosome painting using the chicken $\mathrm{Z}$ chromosome as a probe, gene mapping with a subset of chicken $\mathrm{Z}$ chromosome genes, and in silico analysis have been applied to representative species from 22 reptile families (15 lizards, 3 turtles, 3 snakes and 1 crocodilian). These studies revealed non-homology of sex chromosomes between chicken and reptiles (Ezaz et al. 2009a; Pokorna et al. 2011; www. ensembl.org). The chicken $\mathrm{Z}$ chromosome is homologous over a substantial region of chromosome 2 in representative species from the majority of the families tested $(12 / 22)$, suggesting conservation of this chromosome over a long

Table 4 Reptile species with molecular cytogenetic maps

\begin{tabular}{|c|c|c|c|c|}
\hline Species & Common name & Marker type & No. of markers & Reference \\
\hline A. carolinensis & Green anole lizard & $\mathrm{BAC}$ & 356 & Alfoldi et al. 2011 \\
\hline Elpaphe quadrivirgata & Japanese four-striped rat snake & cDNA & 183 & $\begin{array}{l}\text { Matsubara et al. 2012; Matsubara } \\
\text { et al. 2006; Matsuda et al. } 2005\end{array}$ \\
\hline Pelodiscus sinensis & Chinese soft-shelled turtle & cDNA & 162 & Uno et al. 2012; Matsuda et al. 2005 \\
\hline Crocodylus siamensis & Siamese crocodile & cDNA & 131 & Uno et al. 2012 \\
\hline Pogona vitticeps & Central bearded dragon & BAC & 87 & Young et al. 2013 \\
\hline Varanus salvator macromaculatusi & Water monitor lizard & cDNA & 86 & Srikulnath et al. 2013 \\
\hline Leiolepis reevesii rubritaeniata & Butterfly lizard & cDNA & 54 & Srikulnath et al. 2009 \\
\hline Sphenodon punctatus & Tuatara & $\mathrm{BAC}$ & 21 & O’Meally et al. 2009 \\
\hline Varanus exanthematicus & Savannah monitor lizard & cDNA & 17 & Srikulnath et al. 2013 \\
\hline
\end{tabular}


evolutionary time frame (Ezaz et al. 2009a; Pokorna et al. 2011), albeit none of these are sex chromosomes. However, in species from several families of Gekkota (Gekkonidae, Diplodactylidae, Eublepharidae and Pygopodidae), probes for the chicken $\mathrm{Z}$ chromosome revealed quite an unusual scenario. For example, five genes from the chicken $\mathrm{Z}$ chromosome (ACO1/IREBP-RPS6-DMRT1-CHD1-GHR$A T P 5 A 1)$ are present in the same order on the ZW sex chromosomes of the Hoku gecko, Gekko hokouensis (Kawai et al. 2009), implying that either chicken and G. hokouensis sex chromosomes have evolved from a common ancestor or such homology is a result of convergent evolution via chromosome rearrangements in this species. However, for species from three other families of Gekkota (Diplodactylidae, Eublepharidae and Pygopodidae) and three other lizard families (Angiudae, Lacerdiae and Teiidae), painting of the chicken $\mathrm{Z}$ chromosome showed homology with a medium to small size acrocentric or subtelocentric autosome, not with their sex chromosomes (where present) (Pokorna et al. 2011). Chicken $\mathrm{Z}$ chromosome painting also showed homology to chromosome 6 in two turtle species (Trachemys scripta, Emydidae and $P$. sinensis, Trionychidae), and a crocodile species tested (Crocodylus niloticus, Crocodyldae). It is clear from the above discussion that, although autosomal, the chicken $\mathrm{Z}$ chromosome has retained conserved synteny in the majority of reptiles, with the exception of Gekkonidae, where substantial genomic rearrangements may have contributed in an ancestral syntenic break, including coincidental homology between the sex chromosomes of G. hokouensis and chicken.

In several recent studies, reptile sex chromosome genes have also been used for cross-species gene mapping. For example, four genes from the $\mathrm{Z}$ chromosome of $G$. hokouensis are autosomal in the dragon lizard $P$. vitticeps and five genes from $\mathrm{Z}$ chromosomes of snakes and birds are autosomal in the dragon lizard $P$. vitticeps (Ezaz et al. 2009a). Two genes from ZW sex chromosomes of $P$. vitticeps (Ezaz et al. 2013; Young et al. 2013) and five genes from the XY sex chromosomes of marsh black turtle Siebenrockiella crassicollis are also autosomal in chicken (Kawagoshi et al. 2012). In addition, an Xlinked BAC clone from the green anole is on chicken chromosome 15 (Alfoldi et al. 2011). Such non-homology between avian and reptile sex chromosomes implies multiple and independent origins of sex chromosomes within reptiles, possibly via de novo evolution of sex chromosomes and sex determining genes. However, the de novo evolution can only be verified through targeted studies in those groups with robust phylogeny and evidence of multiple evolution of sex chromosomes within and between species, genera and populations such as in Agamidae and Gekkonidae. A recent in silico comparative study of snake sex chromosomes by Vicoso et al. (2013) presents an excellent example of novel discoveries using genomic and transcriptomic information in a comparative context to understand sex chromosome evolution, degeneration and dosage compensation. This study also provided a platform for comparative analysis of reptilian sex chromosomes, not only with other reptiles but with vertebrates in general.

\section{Mammalian ancestral karyotype reconstructions}

Understanding the events that have led to the diversity observed for mammalian karyotypes requires comparisons to be made across the three major lineages. However, in the study of mammal chromosome evolution, most effort has been placed into determining how the genomes of the karyotypically diverse eutherian mammals have changed since their radiation approximately105 MYA. This is because comparisons with the other two mammalian lineages were impossible using cross-species chromosome painting (Graphodatsky et al. 2012). As a consequence, most studies have focused on reconstructing the eutherian or boreoeutherian ancestral karyotype, rather than on that of the ancestor to all mammals.

Numerous studies have used cross-species chromosome painting to characterize chromosome homology broadly across the eutherian phylogeny as well as in specific eutherian lineages, with these comparisons enabling the reconstruction of the boreoeutherian ancestor (ancestor of most eutherians) or even the ancestral karyotype of all eutherian mammals. Based on cross-species chromosome painting, predominantly with probes to human chromosomes, the genomes of most eutherian species can be divided into 30 to 40 conserved segments (Ferguson-Smith and Trifonov 2007). Exceptions to this level of conservation are observed in species such as dogs and the small apes (gibbons), where the 22 probes to human autosomes detected 73 (Yang et al. 1999) and 51 (Jauch et al. 1992) conserved segments respectively. Overall, chromosome painting with human chromosome probes across the eutherian phylogeny have identified a number of conserved segments corresponding to regions from two or more human chromosomes that are associated in diverse taxa and may therefore, represent an ancestral organization. Several different predicted ancestral eutherian karyotypes have been put forward from these types of analyses, with diploid numbers ranging from 44 to 50 chromosomes (Svartman et al. 2004).

The availability of well-assembled and anchored genomes for diverse eutherian species has added a greater level of confidence to the predicted ancestral karyotypes, particularly when compared to sequenced outgroup species, such as a marsupial, the South American grey short-tailed opossum (Monodelphis domestica), and chicken. Such comparisons have shown that some of the associated segments revealed by chromosome painting amongst eutherian species represent an ancestral organization present in marsupials and even in chicken and the green anole. These associations include the association of segments from human chromosomes 4/8, 12/ 
22, 14/15 and 16/19 (Graphodatsky et al. 2012). The initial attempts made to reconstruct ancestral karyotypes purely from genome sequence comparisons for just a few eutherian species and using chicken as an outgroup, resulted in 'mammalian' ancestral karyotypes consisting of $2 n=42$ (Bourque et al. 2005; International Chicken Genome Sequencing C 2004). The number of adequately assembled and anchored genomes available is one of the major limitations of these types of reconstructions. As more genome sequences have been released, the reconstruction of a putative ancestral eutherian karyotype based on sequence from five eutherian species, opossum and chicken has converged with the widely accepted ancestral eutherian $2 n=46$ karyotype (Kemkemer et al. 2009), similar to the one depicted in Fig. 3. In-depth comparisons of eutherian ancestral reconstructions have been recently reviewed elsewhere and should be referred to for further insight into how the generally accepted $2 n=46$ eutherian karyotype was derived and the supporting evidence (Ferguson-Smith and Trifonov 2007; Graphodatsky et al. 2012; Ruiz-Herrera et al. 2012)

The challenge for more broadly understanding the evolution of mammalian chromosomes now lies with the other two major lineages, the marsupials and monotremes, which diverged from eutherians about 160 (Luo et al. 2011) and 180 MYA (Phillips et al. 2009), respectively. The deep divergence of these three mammalian lineages and the marked differences in their karyotypic features will undoubtedly provide great insight into the events shaping their genomes and the reconstruction of ancestral karyotypes (Deakin et al. 2012b).

\section{Reconstruction of marsupial ancestral karyotype}

Marsupials are renowned for their low diploid chromosome numbers and a high level of conservation across the marsupial phylogeny. The characteristically large chromosomes of marsupials have facilitated cytogenetic studies, with over $70 \%$ of the approximately 330 extant marsupial species karyotyped to date (Hayman 1990). Cross-species chromosome painting using probes derived from the rufous bettong (Aepyprymnus rufescens), the marsupial with the highest diploid number of $2 n=32$, has divided marsupial genomes into just 19 conserved segments (Rens et al. 2003). The speciose Family Dasyuridae show an astonishing level of conservation, as all members of this family karyotyped to date (40 of the 68 known species) have a $2 n=14$ karyotype (Hayman and Martin 1974a; Rofe and Hayman 1985; Young et al. 1982).

Indeed, marsupial karyotyping studies have reported the predominance of this diploid number across marsupial phylogeny, as well as a $2 n=22$ chromosome complement. This led to hypotheses for either one of these diploid numbers representing that of the ancestral marsupial (Matthey 1972). These hypotheses were further developed as more cytogenetic information became available. The $2 n=14$ marsupial ancestor hypothesis is based on this conserved complement being present in six of the seven extant marsupial orders and proposes that fissions of this ancestral karyotype gave rise the higher diploid numbers frequently observed in many marsupial families (Hayman and Martin 1969, 1974a, b; Reig et al. 1977; Rofe and Hayman 1985). The hypothesis for an ancestor with the higher diploid number of $2 n=22$ proposes that the common $2 n=14$ karyotype was derived from fusion events early on in the divergence of the different marsupial lineages (Sharman 1973; Svartman and Vianna-Morgante 1998).

Resolving which one of these hypotheses is more likely has been difficult, as evidence to support both hypotheses has been reported over the last 40 years. Support for the $2 n=14$ hypothesis was substantial, with conservation of the $2 n=14$ karyotype observed by G-banding (Rofe and Hayman 1985) and cross-species chromosome painting across different families of marsupials (De Leo et al. 1999; Rens et al. 2003; Rens et al. 2001), and a well-resolved phylogenetic tree positioning a $2 n=14$ species as the basal species (Westerman et al. 2010). Evidence for the alternative hypothesis was weaker. Crossspecies chromosome painting demonstrated that, despite the commonality of the $2 n=22$ diploid number across different marsupial families, the 18 autosomal segments detected were arranged differently between $2 \mathrm{n}=22$ species (O'Neill et al. 1999; Rens et al. 2003). The main evidence to support the $2 n=$ 22 hypothesis was the observation of interstitial telomere signals in members of the Family Didelphidae, a family representing one of the earliest offshoots of the marsupial lineage, with $2 n=14$ or $2 n=18$ karyotypes, suggesting that chromosome fusions were responsible for these lower diploid numbers (Carvalho and Mattevi 2000; Svartman and ViannaMorgante 1998). The inability to compare marsupial chromosome arrangement to outgroup species using chromosome painting prevented further testing of either hypothesis. However, this limitation was recently overcome with the availability of the well assembled and anchored opossum (M. domestica) genome (Duke et al. 2007; Mikkelsen et al. 2007) and the sequence and cytogenetic map for the tammar wallaby (Macropus eugenii) genome (Deakin et al. 2013; Renfree et al. 2011), enabling comparisons to be made to outgroups such as human and chicken (Deakin et al. 2013; Deakin et al. 2012b).

Comparison of gene arrangement between the two marsupial species and two outgroup species permitted the ancestral marsupial karyotype to be reconstructed, supporting the $2 n=14$ hypothesis. The arrangement of the conserved segments in the $2 n=14$ species was observed to be more ancestral than that predicted for the $2 n=22$ species. The chromosome arrangements of other species could be easily derived, mainly by fissions and inversions, from a $2 n=14$ karyotype (Deakin et al. 2013).

Although there is strong evidence for a $2 n=14$ ancestral karyotype, the presence of interstitial telomere signals on 
Fig. 3 Predicted ancestral karyotypes for mammals, colourcoded for homology to human chromosomes

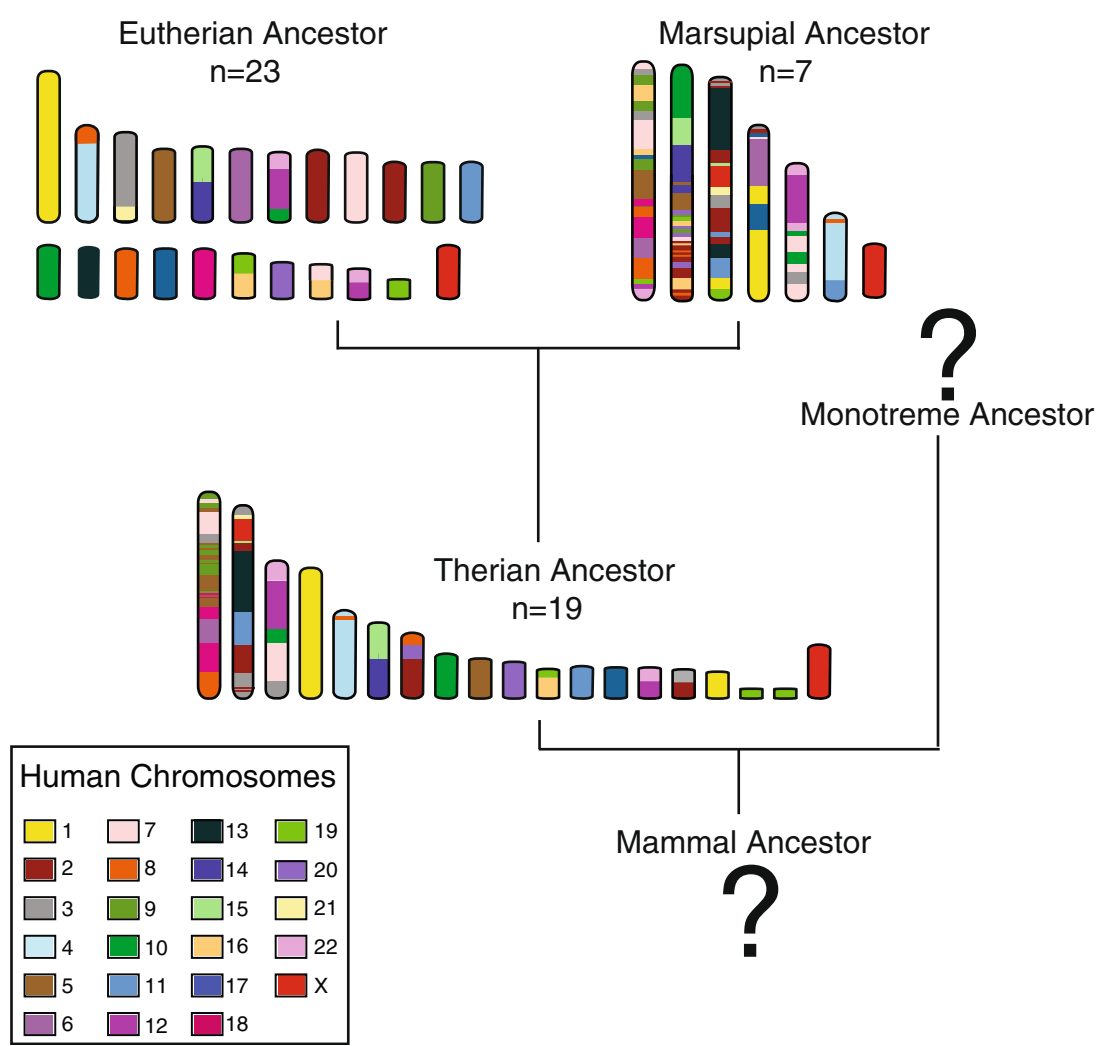

M. domestica chromosome still needs to be explained. These signals are thought to be the result of a centric fusion but they may actually represent satellite DNA, as C-banding has shown that these signals coincide with pericentric heterochromatin (Pagnozzi et al. 2002). Furthermore, interstitial telomere signals have been observed on marsupial chromosomes that would not have been formed from fusion events from either a $2 n=14$ or $2 n=22$ marsupial ancestor. For example, in the $2 n=14$ fat-tailed dunnart, interstitial telomere signals are observed on chromosome 6 but this chromosome is homologous to an entire chromosome in the $2 n=22$ common opossum and in a predicted $2 n=22$ ancestor. This chromosome, however, appears to have undergone at least a couple of large-scale inversions which could have moved telomeric sequences to an interstitial location (Deakin et al. 2012b). Likewise, an inversion event may have occurred in $M$. domestica. Information on gene order for more marsupial species, particularly Didelphis marsupialis would help to test this idea.

Marsupial chromosomes, when compared by the broad brushstrokes of chromosome painting, may appear to be well conserved but, like bird chromosomes, they have been prone to intrachromosomal rearrangements. A comparison of the opossum genome assembly with the tammar wallaby gene map (Deakin et al. 2013; Deakin et al. 2008), consisting of 554 genes, and the devil gene map consisting of 105 (Deakin et al. 2012a) genes, makes it clear that certain chromosomes have undergone extensive rearrangement since these three species last shared a common ancestor about 80 million years ago. These rearrangements mainly appear to be the result of inversions or a series of inversions, particularly involving conserved segments C1, C2 and C3 (Fig. 4). The 18 conserved segments identified by chromosome painting on marsupial autosomes translated into 76 segments when the tammar wallaby gene map was compared to the opossum genome (Deakin et al. 2013). At this stage, it is unclear how many conserved segments are present when the more lightly mapped devil genome is compared to that of the tammar wallaby or opossum. Mapping of more genes and attempts to improve the genome assembly are currently being carried for more in-depth comparisons.

Analysis of the associations of chromosome segments observed in marsupials and the predicted arrangement in the ancestral marsupial described above, combined with comparisons to the chicken as an outgroup species, has brought us a step closer to deciphering the ancestral karyotype of therian mammals, something that has been difficult to do without the detailed information for more than one marsupial. The predicted ancestral therian karyotype has a $2 n=19$ chromosome complement (Fig. 3), from which the ancestral marsupial karyotype can be derived by fusion events and the ancestral eutherian karyotype derived from inversions, fissions and fusions (Deakin et al. 2013). This has brought us a step closer to reconstructing the ancestor of all mammals but which ultimately requires information from monotreme genomes (discussed below). 


\section{$\mathrm{X}$ chromosomes of therian mammals}

Mammals have an XX/XY sex chromosome system. The X chromosome is well conserved in gene content between marsupials and eutherians, with about two thirds of the eutherian $\mathrm{X}$ sharing homology with the $\mathrm{X}$ of marsupials (Glas et al. 1999). There has been an addition to the $X$ chromosome in the common ancestor of all eutherians (Graves 1995). With the exception of rodents, gene order is remarkably well conserved amongst eutherians, even between humans and the African elephant (Delgado et al. 2009), a representative of the most basal eutherian lineage, the Afrotheria. This is thought to be a consequence of $\mathrm{X}$ chromosome inactivation, part of the dosage compensation mechanism to equalize X-borne gene expression between males and females, where rearrangements of the $\mathrm{X}$ chromosome may risk disrupting this complex mechanism (Mikkelsen et al. 2007). In contrast, gene order is not conserved between marsupials and eutherians or even between different marsupial species (Deakin et al. 2008). Although marsupials also inactivate one X chromosome in female somatic cells, the mechanism is quite different from that observed in eutherians mammals (reviewed in Deakin 2013) and may be more tolerant of intrachromosomal rearrangements.

The challenges presented by monotreme genomes

Monotremes, as the earliest offshoot of the mammalian lineage, occupy an important position in amniote phylogeny for the reconstruction of the karyotype for the therian ancestor and the ancestor to all mammals. This lineage consists of just one species of platypus (Ornithorhynchus anatinus) and four species of echidna. Monotreme karyotypes feature several large chromosomes and a large number of smaller chromosomes that could not be conclusively distinguished by banding patterns (Wrigley and Graves 1988a, b). It was only when chromosome painting with these small chromosomes was carried out that it became clear that some of them represented different X and Y chromosomes (Rens et al. 2004; Grutzner et al. 2004). Astonishingly, these sex chromosomes form an alternating $\mathrm{X}$ and $\mathrm{Y}$ chain during male meiosis (Grutzner et al. 2004), and share no gene content with that of the ancestral therian $\mathrm{X}$ chromosome, but instead share homology with the $\mathrm{Z}$ chromosome of birds (Veyrunes et al. 2008).

Comparisons between platypus and echidna chromosomes have revealed extensive rearrangement between these species. Chromosome painting has shown that platypus chromosomes $1,4,5,9,11,14,16$ and 19 correspond to echidna chromosomes $1,4,3,10,11,14,19$ and 22, respectively but all other chromosomes are derived, mainly by Robertsonian fissions and fusions (Rens et al. 2007). There are even differences in the composition of the sex chromosomes between platypus

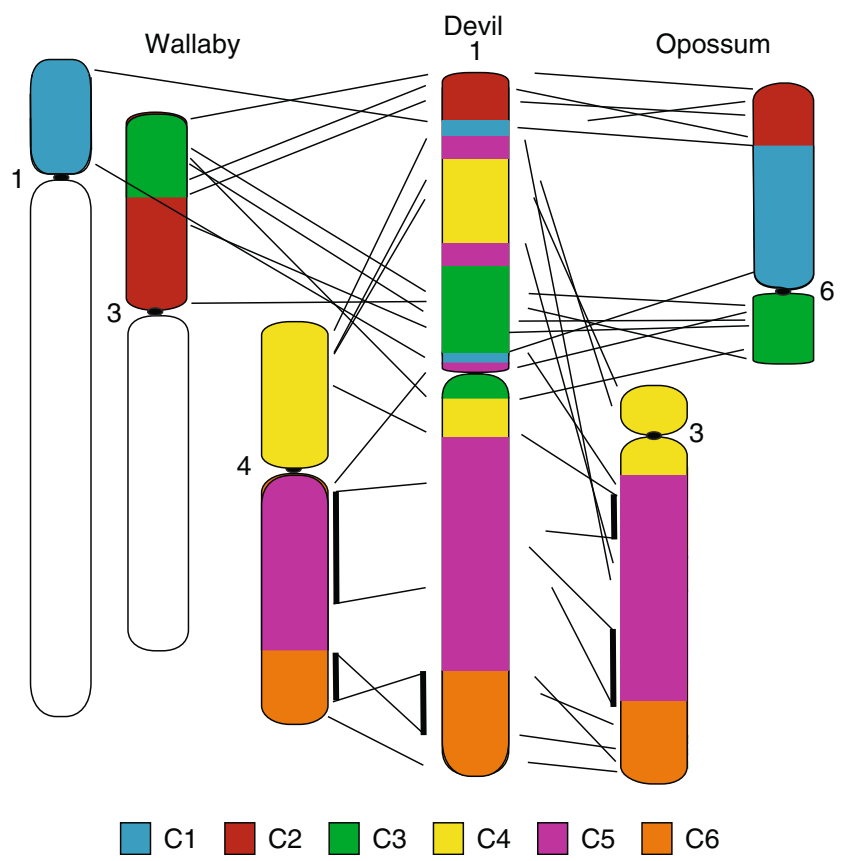

Fig. 4 Comparison of gene arrangement between the tammar wallaby, Tasmanian devil and grey short-tailed opossum for conserved segments C1 to C6. Modified from Deakin et al. (2012a, b)

and echidna. Male platypuses have five X and five $\mathrm{Y}$ chromosomes but the short-beaked echidna (Tachyglossus aculeatus) has five $\mathrm{X}$ chromosomes and only four $\mathrm{Y}$ chromosomes. $\mathrm{X}_{1}, \mathrm{X}_{2}$ and $\mathrm{X}_{3}$ are homologous between these two species, however, $X_{4}$ in platypus is homologous to echidna chromosome 27 and echidna $\mathrm{X}_{5}$ shares homology with platypus $12 \mathrm{p}$. Reconstructing the ancestral monotreme genome is difficult given that there are so few species in this lineage and the difference between karyotypes (Deakin et al. 2012b; 2013).

To date, monotremes have failed to live up to their potential for aiding in the reconstruction of the therian and mammalian ancestral karyotypes due to several factors. Firstly, as mentioned previously, chromosome paints from other mammalian lineages do not hybridise to monotreme chromosomes (Graphodatsky et al. 2012). Secondly, although the platypus genome has been sequenced, only about a fifth of this sequence has been anchored to chromosomes (Warren et al. 2008), preventing the karyotype of the ancestor to all mammals to be reconstructed. This is largely due to abundant repetitive sequences making it challenging to assemble the genome sequence (Warren et al. 2008). Despite this, the platypus genome assembly has provided some evidence for segmental associations (4q/8q, 12qter/22q, 7a/16p, 3/21, 16q/ $19 q$ and $22 q 12 / 12 q 24.3)$ conserved across the Class Mammalia, but this may not be a complete list of all those that are present (Ruiz-Herrera et al. 2012).

Despite the insufficiencies of the platypus genome assembly, as mentioned previously, a therian ancestral karyotype has 
been predicted (Fig. 3). This prediction obviously has its limitations and having a well-anchored monotreme genome assembly would have provided a greater degree of confidence in the reconstruction (Deakin et al. 2013). Hopefully, continued efforts in monotreme and marsupial genomics will make it possible to test this reconstructed karyotype in the future.

\section{Conclusions and future directions}

Genome technology is making this an exciting time to be exploring the evolution of amniote genomes. However, it is hoped that data from key species in different amniote lineages will help to either confirm current predicted ancestral chromosome arrangements or provide the much needed insight into their reconstruction. For squamate reptiles, it will be appropriate to develop a denser physical map for $P$. vitticeps, a species which already has a preliminary physical map, BAC library and whole genome sequence assembly at a draft stage. This will represent a ZW species, while the green anole genome represents an XY squamate lizard. The tuatara genome is currently being sequenced and the development of a physical map would be appropriate for this lone representative of living Sphenodont. Ideally, at least one species from each major reptilian lineage should have a genome map. Whole genome sequence data from some of the representative groups is already available (e.g. python, king cobra, three species of turtle) so it would be sensible to produce maps for these species. Monotremes are situated at a particularly important phylogenetic location. The echidna genome is also currently being sequenced, yet without being anchored to chromosomes, it will be of little value for studies into genome evolution. It is to be hoped that either a physical map will be produced or that the advances in genome sequencing technology will improve monotreme genome assemblies.

An exciting area of research that needs to be further developed in regards to amniote chromosome evolution is the functional relevance of regions conserved as HSBs and the sequence features contributing to chromosomal rearrangements. For instance, large HSBs conserved across eight eutherians, opossum and chicken are enriched in genes important for development, particularly of the central nervous system and have most likely been conserved as a block to avoid disrupting the important combinations of genes and regulatory elements. In contrast, evolutionary breakpoint regions (EBRs) are commonly enriched for genes related to an organism's response to external stimuli (Larkin et al. 2009). This also highlights the critical requirement of more anchored amniote genome assemblies for ultimately determining the consequence of chromosomal rearrangements on gene regulation and function that have led to the major phenotypic differences between the different amniote lineages.
Acknowledgments JED and TE are supported by Australian Research Council Future Fellowships (FT100100241 and FT110100733).

Open Access This article is distributed under the terms of the Creative Commons Attribution License which permits any use, distribution, and reproduction in any medium, provided the original author(s) and the source are credited.

\section{References}

Adolfsson S, Ellegren H (2013) Lack of dosage compensation accompanies the arrested stage of sex chromosome evolution in ostriches. Mol Biol Evol 30(4):806-810. doi:10.1093/molbev/mst009

Alfoldi J, Di Palma F, Grabherr M, Williams C, Kong L, Mauceli E et al (2011) The genome of the green anole lizard and a comparative analysis with birds and mammals. Nature 477(7366):587-591. doi: 10.1038 /nature 10390

Axelsson E, Webster MT, Smith NG, Burt DW, Ellegren H (2005) Comparison of the chicken and turkey genomes reveals a higher rate of nucleotide divergence on microchromosomes than macrochromosomes. Genome Res 15(1):120-125. doi:10.1101/gr. 3021305

Bourque G, Zdobnov EM, Bork P, Pevzner PA, Tesler G (2005) Comparative architectures of mammalian and chicken genomes reveal highly variable rates of genomic rearrangements across different lineages. Genome Res 15(1):98-110. doi:10.1101/ gr.3002305

Burt DW (2002) Origin and evolution of avian microchromosomes. Cytogenet Genome Res 96(1-4):97-112. doi:10.1093/molbev/ mst009

Carvalho BD, Mattevi MS (2000) (T2AG3)n telomeric sequence hybridization suggestive of centric fusion in karyotype marsupials evolution. Genetica 108(3):205-210

Castoe TA, de Koning AP, Hall KT, Card DC, Schield DR, Fujita MK et al (2013) The Burmese python genome reveals the molecular basis for extreme adaptation in snakes. Proc Natl Acad Sci U S A 110(51):20645-20650. doi:10.1073/pnas.1314475110

Chen N, Bellott DW, Page DC, Clark AG (2012) Identification of avian W-linked contigs by short-read sequencing. BMC Genomics 13: 183. doi:10.1186/1471-2164-13-183

Christidis L (1990) Animal cytogenetics 4: Chordata 3 B: Aves. Gebruder Borntraeger, Berlin

Cohen MM, Gans C (1970) The chromosomes of the order Crocodilia. Cytogenetics 9(2):81-105

Dalloul RA, Long JA, Zimin AV, Aslam L, Beal K, Blomberg Le A et al. (2010) Multi-platform next-generation sequencing of the domestic turkey (Meleagris gallopavo): genome assembly and analysis. PLoS Biol 8 (9). doi:10.1371/journal.pbio.1000475

De Leo AA, Guedelha N, Toder R, Voullaire L, Ferguson-Smith MA, O'Brien PC, Graves JA (1999) Comparative chromosome painting between marsupial orders: relationships with a $2 n=14$ ancestral marsupial karyotype. Chromosom Res 7(7):509-517

de Oliveira EH, Habermann FA, Lacerda O, Sbalqueiro IJ, Wienberg J, Muller S (2005) Chromosome reshuffling in birds of prey: the karyotype of the world's largest eagle (Harpy eagle, Harpia harpyja) compared to that of the chicken (Gallus gallus). Chromosoma 114(5):338-343. doi:10.1007/s00412-005-0009-5

de Oliveira EH, de Moura SP, dos Anjos LJ, Nagamachi CY, Pieczarka JC, O'Brien PC, Ferguson-Smith MA (2008) Comparative chromosome painting between chicken and spectacled owl (Pulsatrix perspicillata): implications for chromosomal evolution in the Strigidae (Aves, Strigiformes). Cytogenet Genome Res 122(2): 157-162. doi:10.1159/000163093 
Deakin JE (2013) Marsupial X chromosome inactivation: past, present and future. Aust J Zool 61(1):13-23. doi:10.1071/Zo12113

Deakin JE, Koina E, Waters PD, Doherty R, Patel VS, Delbridge ML, Dobson B, Fong J, Hu Y, van den Hurk C, Pask AJ, Shaw G, Smith C, Thompson K, Wakefield MJ, Yu H, Renfree MB, Graves JA (2008) Physical map of two tammar wallaby chromosomes: a strategy for mapping in non-model mammals. Chromosom Res 16(8): 1159-1175. doi:10.1007/s10577-008-1266-y

Deakin JE, Bender HS, Pearse AM, Rens W, O'Brien PC, FergusonSmith MA, Cheng Y, Morris K, Taylor R, Stuart A, Belov K, Amemiya CT, Murchison EP, Papenfuss AT, Graves JA (2012a) Genomic restructuring in the Tasmanian devil facial tumour: chromosome painting and gene mapping provide clues to evolution of a transmissible tumour. PLoS Genet 8(2):e1002483. doi:10.1371/ journal.pgen.1002483

Deakin JE, Graves JA, Rens W (2012b) The evolution of marsupial and monotreme chromosomes. Cytogenet Genome Res 137(2-4):113129. doi:10.1159/000339433

Deakin JE, Delbridge ML, Koina E, Harley N, Alsop AE, Wang C, Patel VS, Graves JA (2013) Reconstruction of the ancestral marsupial karyotype from comparative gene maps. BMC Evol Biol 13:258. doi:10.1186/1471-2148-13-258

Delgado CL, Waters PD, Gilbert C, Robinson TJ, Graves JA (2009) Physical mapping of the elephant $\mathrm{X}$ chromosome: conservation of gene order over 105 million years. Chromosom Res 17(7):917-926. doi:10.1007/s10577-009-9079-1

Derjusheva S, Kurganova A, Habermann F, Gaginskaya E (2004) High chromosome conservation detected by comparative chromosome painting in chicken, pigeon and passerine birds. Chromosom Res 12(7):715-723. doi:10.1023/B:CHRO.0000045779.50641.00

Duke SE, Samollow PB, Mauceli E, Lindblad-Toh K, Breen M (2007) Integrated cytogenetic BAC map of the genome of the gray, shorttailed opossum, Monodelphis domestica. Chromosom Res 15(3): 361-370. doi:10.1007/s10577-007-1131-4

Ellegren H (2010) Evolutionary stasis: the stable chromosomes of birds. Trends Ecol Evol 25(5):283-291. doi:10.1016/j.tree.2009.12.004

Ellegren H, Smeds L, Burri R, Olason PI, Backstrom N, Kawakami T, Kunstner A, Makinen H, Nadachowska-Brzyska K, Qvarnstrom A, Uebbing S, Wolf JB (2012) The genomic landscape of species divergence in Ficedula flycatchers. Nature 491(7426):756-760. doi:10.1038/nature11584

Ezaz T, Quinn AE, Miura I, Sarre SD, Georges A, Marshall Graves JA (2005) The dragon lizard Pogona vitticeps has ZZ/ZW micro-sex chromosomes. Chromosom Res 13(8):763-776. doi:10.1007/ s10577-005-1010-9

Ezaz T, Moritz B, Waters P, Marshall Graves JA, Georges A, Sarre SD (2009a) The ZW sex microchromosomes of an Australian dragon lizard share no homology with those of other reptiles or birds. Chromosom Res 17(8):965-973. doi:10.1007/s10577-009-9102-6

Ezaz T, Quinn AE, Sarre SD, O’Meally D, Georges A, Graves JA (2009b) Molecular marker suggests rapid changes of sex-determining mechanisms in Australian dragon lizards. Chromosom Res 17(1):91-98. doi:10.1007/s10577-008-9019-5

Ezaz T, Azad B, OM D, Young MJ, Matsubara K, Edwards MJ, Zhang X, Holleley CE, Deakin JE, Marshall Graves JA, Georges A, Edwards SV, Sarre SD (2013) Sequence and gene content of a large fragment of a lizard sex chromosome and evaluation of candidate sex differentiating gene R-spondin 1. BMC Genomics 14(1):899

Ferguson-Smith MA, Trifonov V (2007) Mammalian karyotype evolution. Nat Rev Genet 8(12):950-962. doi:10.1038/nrg2199

Fillon V, Vignoles M, Crooijmans RP, Groenen MA, Zoorob R, Vignal A (2007) FISH mapping of 57 BAC clones reveals strong conservation of synteny between Galliformes and Anseriformes. Anim Genet 38(3):303-307. doi:10.1111/j.1365-2052.2007.01578.x

Giovannotti M, Caputo V, O'Brien PC, Lovell FL, Trifonov V, Cerioni PN, Olmo E, Ferguson-Smith MA, Rens W (2009) Skinks (Reptilia:
Scincidae) have highly conserved karyotypes as revealed by chromosome painting. Cytogenet Genome Res 127(2-4):224-231. doi: $10.1159 / 000295002$

Glas R, Marshall Graves JA, Toder R, Ferguson-Smith M, O'Brien PC (1999) Cross-species chromosome painting between human and marsupial directly demonstrates the ancient region of the mammalian X. Mamm Genome 10(11):1115-1116

Graphodatsky A, Ferguson-Smith MA, Stanyon R (2012) A short introduction to cytogenetic studies in mammals with reference to the present volume. Cytogenet Genome Res 137(2-4):83-96. doi:10. $1159 / 000341502$

Graves JA (1995) The evolution of mammalian sex chromosomes and the origin of sex determining genes. Philos Trans R Soc Lond B Biol Sci 350(1333):305-311. doi:10.1098/rstb.1995.0166, discussion 311302

Griffin DK, Haberman F, Masabanda J, O’Brien P, Bagga M, Sazanov A, Smith J, Burt DW, Ferguson-Smith M, Wienberg J (1999) Microand macrochromosome paints generated by flow cytometry and microdissection: tools for mapping the chicken genome. Cytogenet Cell Genet 87(3-4):278-281

Griffin DK, Robertson LB, Tempest HG, Skinner BM (2007) The evolution of the avian genome as revealed by comparative molecular cytogenetics. Cytogenet Genome Res 117(1-4):64-77. doi:10.1159/ 000103166

Griffin DK, Robertson LB, Tempest HG, Vignal A, Fillon V, Crooijmans RP, Groenen MA, Deryusheva S, Gaginskaya E, Carre W, Waddington D, Talbot R, Volker M, Masabanda JS, Burt DW (2008) Whole genome comparative studies between chicken and turkey and their implications for avian genome evolution. BMC Genomics 9:168. doi:10.1186/1471-2164-9-168

Grutzner F, Zend-Ajusch E, Stout K, Munsche S, Niveleau A, Nanda I, Schmid M, Haaf T (2001) Chicken microchromosomes are hypermethylated and can be identified by specific painting probes. Cytogenet Cell Genet 93(3-4):265-269

Grutzner F, Rens W, Tsend-Ayush E, El-Mogharbel N, O'Brien PC, Jones RC, Ferguson-Smith MA, Marshall Graves JA (2004) In the platypus a meiotic chain of ten sex chromosomes shares genes with the bird Z and mammal X chromosomes. Nature 432(7019):913-917. doi:10.1038/nature03021

Hansmann T, Nanda I, Volobouev V, Yang F, Schartl M, Haaf T, Schmid M (2009) Cross-species chromosome painting corroborates microchromosome fusion during karyotype evolution of birds. Cytogenet Genome Res 126(3):281-304. doi:10.1159/ 000251965

Hayman DL (1990) Marsupial cytogenetics. Aust J Zool 37(2-4):331349

Hayman DL, Martin PG (1969) Cytogenetics of marsupials. In: Benirschke K (ed) Comparative mammalian cytogenetics. Springer, New York, pp 191-217

Hayman DL, Martin PG (1974a) Mammalia I: Monotremata and Marsupialia. In: John B (ed) Animal cytogenetics, vol 4: Chordata 4. Gebruder Borntraeger, Berlin and Stuttgart

Hayman DL, Martin PG (1974b) Marsupial cytogenetics. Gebruder Borntraeger, Berlin

Huang Y, Li Y, Burt DW, Chen H, Zhang Y, Qian W et al (2013) The duck genome and transcriptome provide insight into an avian influenza virus reservoir species. Nat Genet 45(7):776-783. doi:10.1038/ng. 2657

International Chicken Genome Sequencing C (2004) Sequence and comparative analysis of the chicken genome provide unique perspectives on vertebrate evolution. Nature 432(7018):695-716. doi:10.1038/ nature 03154

Jauch A, Wienberg J, Stanyon R, Arnold N, Tofanelli S, Ishida T, Cremer $\mathrm{T}$ (1992) Reconstruction of genomic rearrangements in great apes and gibbons by chromosome painting. Proc Natl Acad Sci U S A 89(18):8611-8615 
Kasai F, O'Brien PC, Martin S, Ferguson-Smith MA (2012) Extensive homology of chicken macrochromosomes in the karyotypes of Trachemys scripta elegans and Crocodylus niloticus revealed by chromosome painting despite long divergence times. Cytogenet Genome Res 136(4):303-307. doi: $10.1159 / 000338111$

Kawagoshi T, Nishida C, Matsuda Y (2012) The origin and differentiation process of $\mathrm{X}$ and $\mathrm{Y}$ chromosomes of the black marsh turtle (Siebenrockiella crassicollis, Geoemydidae, Testudines). Chromosom Res 20(1):95-110. doi:10.1007/s10577-011-9267-7

Kawai A, Ishijima J, Nishida C, Kosaka A, Ota H, Kohno S, Matsuda Y (2009) The ZW sex chromosomes of Gekko hokouensis (Gekkonidae, Squamata) represent highly conserved homology with those of avian species. Chromosoma 118(1):43-51. doi:10.1007/ s00412-008-0176-2

Kayang BB, Fillon V, Inoue-Murayama M, Miwa M, Leroux S, Feve K, Monvoisin JL, Pitel F, Vignoles M, Mouilhayrat C, Beaumont C, Ito S, Minvielle F, Vignal A (2006) Integrated maps in quail (Coturnix japonica) confirm the high degree of synteny conservation with chicken (Gallus gallus) despite 35 million years of divergence. BMC Genomics 7:101. doi:10.1186/1471-2164-7-101

Kemkemer C, Kohn M, Cooper DN, Froenicke L, Hogel J, Hameister H, Kehrer-Sawatzki H (2009) Gene synteny comparisons between different vertebrates provide new insights into breakage and fusion events during mammalian karyotype evolution. BMC Evol Biol 9: 84. doi:10.1186/1471-2148-9-84

Larkin DM, Pape G, Donthu R, Auvil L, Welge M, Lewin HA (2009) Breakpoint regions and homologous synteny blocks in chromosomes have different evolutionary histories. Genome Res 19(5): 770-777. doi:10.1101/gr.086546.108

Lewin HA, Larkin DM, Pontius J, O’Brien SJ (2009) Every genome sequence needs a good map. Genome Res 19(11):1925-1928. doi: 10.1101/gr.094557.109

Luo ZX, Yuan CX, Meng QJ, Ji Q (2011) A Jurassic eutherian mammal and divergence of marsupials and placentals. Nature 476(7361): 442-445. doi:10.1038/nature10291

Matsubara K, Tarui H, Toriba M, Yamada K, Nishida-Umehara C, Agata K, Matsuda Y (2006) Evidence for different origin of sex chromosomes in snakes, birds, and mammals and step-wise differentiation of snake sex chromosomes. Proc Natl Acad Sci U S A 103(48): 18190-18195

Matsubara K, Kuraku S, Tarui H, Nishimura O, Nishida C, Agata K, Kumazawa Y, Matsuda Y (2012) Intra-genomic GC heterogeneity in sauropsids: evolutionary insights from cDNA mapping and GC(3) profiling in snake. BMC Genomics 13:604. doi:10.1186/ 1471-2164-13-604

Matsuda Y, Nishida-Umehara C, Tarui H, Kuroiwa A, Yamada K, Isobe T, Ando J, Fujiwara A, Hirao Y, Nishimura O, Ishijima J, Hayashi A, Saito T, Murakami T, Murakami Y, Kuratani S, Agata K (2005) Highly conserved linkage homology between birds and turtles: bird and turtle chromosomes are precise counterparts of each other. Chromosom Res 13(6):601-615. doi:10.1007/s10577-005-0986-5

Matthey R (1949) Les chromosomes des vertebrees. Rouge, Lucerne

Matthey R (1972) The chromosome formulae of eutherian mammals. In: Chiarelli R, Cappanna E (eds) Cytotaxonomy and vertebrate evolution. Academic, London, pp 531-553

Mengden GA, Stock AD (1980) Chromosomal Evolution in Serpentesa Comparison of Chromosome-G and Chromosome-C BandingPatterns of Some Colubrid and Boid Genera. Chromosoma 79(1): 53-64. doi:10.1007/Bf00328472

Mikkelsen TS, Wakefield MJ, Aken B, Amemiya CT, Chang JL, Duke S et al (2007) Genome of the marsupial Monodelphis domestica reveals innovation in non-coding sequences. Nature 447(7141): 167-177. doi:10.1038/nature 05805

Nanda I, Karl E, Griffin DK, Schartl M, Schmid M (2007) Chromosome repatterning in three representative parrots (Psittaciformes) inferred from comparative chromosome painting. Cytogenet Genome Res 117(1-4):43-53. doi:10.1159/000103164

Nanda I, Benisch P, Fetting D, Haaf T, Schmid M (2011) Synteny conservation of chicken macrochromosomes 1-10 in different avian lineages revealed by cross-species chromosome painting. Cytogenet Genome Res 132(3):165-181. doi:10.1159/000322358

Nie W, O'Brien PC, Ng BL, Fu B, Volobouev V, Carter NP, FergusonSmith MA, Yang F (2009) Avian comparative genomics: reciprocal chromosome painting between domestic chicken (Gallus gallus) and the stone curlew (Burhinus oedicnemus, Charadriiformes) - an atypical species with low diploid number. Chromosom Res 17(1): 99-113. doi:10.1007/s10577-009-9021-6

Nishida C, Ishijima J, Kosaka A, Tanabe H, Habermann FA, Griffin DK, Matsuda Y (2008) Characterization of chromosome structures of Falconinae (Falconidae, Falconiformes, Aves) by chromosome painting and delineation of chromosome rearrangements during their differentiation. Chromosom Res 16(1):171-181. doi:10.1007/ s10577-007-1210-6

Nishida C, Ishijima J, Ishishita S, Yamada K, Griffin DK, Yamazaki T, Matsuda Y (2013) Karyotype reorganization with conserved genomic compartmentalization in dot-shaped microchromosomes in the Japanese mountain hawk-eagle (Nisaetus nipalensis orientalis, Accipitridae). Cytogenet Genome Res 141(4):284-294. doi:10. $1159 / 000352067$

Nishida-Umehara C, Tsuda Y, Ishijima J, Ando J, Fujiwara A, Matsuda Y, Griffin DK (2007) The molecular basis of chromosome orthologies and sex chromosomal differentiation in palaeognathous birds. Chromosom Res 15(6):721-734. doi:10.1007/s10577-007-1157-7

O'Brien SJ, Menninger JC, Nash WG (2006) Atlas of mammalian chromosomes. Wiley, New York

Oleksyk TK, Pombert JF, Siu D, Mazo-Vargas A, Ramos B, Guiblet W, Afanador Y, Ruiz-Rodriguez CT, Nickerson ML, Logue DM, Dean M, Figueroa L, Valentin R, Martinez-Cruzado JC (2012) A locally funded Puerto Rican parrot (Amazona vittata) genome sequencing project increases avian data and advances young researcher education. Gigascience 1(1):14. doi:10.1186/2047-217X-1-14

Olmo E (2008) Trends in the evolution of reptilian chromosomes. Integr Comp Biol 48(4):486-493. doi:10.1093/icb/icn049

Olmo E, Signorino G (2005) Chromorep: a reptiles chromosomes database. http://chromorep.univpm.it/. Accessed December 2013

O'Meally D, Miller H, Patel HR, Graves JA, Ezaz T (2009) The first cytogenetic map of the tuatara, Sphenodon punctatus. Cytogenet Genome Res 127(2-4):213-223. doi:10.1159/000300099

O’Meally D, Patel HR, Stiglec R, Sarre SD, Georges A, Marshall Graves JA, Ezaz T (2010) Non-homologous sex chromosomes of birds and snakes share repetitive sequences. Chromosom Res 18(7):787-800. doi:10.1007/s10577-010-9152-9

O'Neill RJ, Eldridge MD, Toder R, Ferguson-Smith MA, O'Brien PC, Graves JA (1999) Chromosome evolution in kangaroos (Marsupialia: Macropodidae): cross species chromosome painting between the tammar wallaby and rock wallaby spp. with the $2 n=22$ ancestral macropodid karyotype. Genome 42(3):525-530

Pagnozzi JM, Ditchfield AD, Yonenaga-Yassuda Y (2002) Mapping the distribution of the interstitial telomeric (TTAGGG)n sequences in eight species of Brazilian marsupials (Didelphidae) by FISH and the correlation with constitutive heterochromatin. Do ITS represent evidence for fusion events in American marsupials? Cytogenet Genome Res 98(4):278-284. doi:10.1159/000071049

Phillips MJ, Bennett TH, Lee MS (2009) Molecules, morphology, and ecology indicate a recent, amphibious ancestry for echidnas. Proc Natl Acad Sci U S A 106(40):17089-17094. doi:10.1073/pnas. 0904649106

Pigozzi MI, Solari AJ (1997) Extreme axial equalization and wide distribution of recombination nodules in the primitive $\mathrm{ZW}$ pair of Rhea americana (Aves, Ratitae). Chromosom Res 5(6):421-428 
Pokorna M, Giovannotti M, Kratochvil L, Kasai F, Trifonov VA, O’Brien PC, Caputo V, Olmo E, Ferguson-Smith MA, Rens W (2011) Strong conservation of the bird $\mathrm{Z}$ chromosome in reptilian genomes is revealed by comparative painting despite 275 million years divergence. Chromosoma 120(5):455-468. doi:10.1007/s00412-0110322-0

Pokorna M, Giovannotti M, Kratochvil L, Caputo V, Olmo E, FergusonSmith MA, Rens W (2012) Conservation of chromosomes syntenic with avian autosomes in squamate reptiles revealed by comparative chromosome painting. Chromosoma 121(4):409-418. doi:10.1007/ s00412-012-0371-z

Rands CM, Darling A, Fujita M, Kong L, Webster MT, Clabaut C, Emes RD, Heger A, Meader S, Hawkins MB, Eisen MB, Teiling C, Affourtit J, Boese B, Grant PR, Grant BR, Eisen JA, Abzhanov A, Ponting CP (2013) Insights into the evolution of Darwin's finches from comparative analysis of the Geospiza magnirostris genome sequence. BMC Genomics 14:95. doi:10.1186/1471-2164-14-95

Reig OA, Gardner AL, Bianchi NO, Patton JL (1977) The chromosomes of the Didelphidae (Marsupialia) and their evolutionary significance. Biol J Linn Soc 9:191-216

Renfree MB, Papenfuss AT, Deakin JE, Lindsay J, Heider T, Belov K et al (2011) Genome sequence of an Australian kangaroo, Macropus eugenii, provides insight into the evolution of mammalian reproduction and development. Genome Biol 12(8):R81. doi:10.1186/gb2011-12-8-r81

Rens W, O'Brien PC, Yang F, Solanky N, Perelman P, Graphodatsky AS, Ferguson MW, Svartman M, De Leo AA, Graves JA, FergusonSmith MA (2001) Karyotype relationships between distantly related marsupials from South America and Australia. Chromosom Res 9(4):301-308

Rens W, O'Brien PC, Fairclough H, Harman L, Graves JA, FergusonSmith MA (2003) Reversal and convergence in marsupial chromosome evolution. Cytogenet Genome Res 102(1-4):282-290. doi:10. $1159 / 000075764$

Rens W, Grutzner F, O'Brien PC, Fairclough H, Graves JA, FergusonSmith MA (2004) Resolution and evolution of the duck-billed platypus karyotype with an X1Y1X2Y2X3Y3X4Y4X5Y5 male sex chromosome constitution. Proc Natl Acad Sci U S A 101(46): 16257-16261. doi:10.1073/pnas.0405702101

Rens W, O'Brien PC, Grutzner F, Clarke O, Graphodatskaya D, TsendAyush E, Trifonov VA, Skelton H, Wallis MC, Johnston S, Veyrunes F, Graves JA, Ferguson-Smith MA (2007) The multiple sex chromosomes of platypus and echidna are not completely identical and several share homology with the avian Z. Genome Biol 8(11):R243. doi:10.1186/gb-2007-8-11-r243

Rofe R, Hayman D (1985) G-banding evidence for a conserved complement in the Marsupialia. Cytogenet Cell Genet 39(1):40-50

Ruiz-Herrera A, Farre M, Robinson TJ (2012) Molecular cytogenetic and genomic insights into chromosomal evolution. Heredity (Edinb) 108(1):28-36. doi:10.1038/hdy.2011.102

Seabury CM, Dowd SE, Seabury PM, Raudsepp T, Brightsmith DJ, Liboriussen P, Halley Y, Fisher CA, Owens E, Viswanathan G, Tizard IR (2013) A multi-platform draft de novo genome assembly and comparative analysis for the Scarlet Macaw (Ara macao). PLoS One 8(5):e62415. doi:10.1371/journal.pone.0062415

Shaffer HB, Minx P, Warren DE, Shedlock AM, Thomson RC, Valenzuela $N$ et al (2013) The western painted turtle genome, a model for the evolution of extreme physiological adaptations in a slowly evolving lineage. Genome Biol 14(3):R28. doi:10.1186/gb2013-14-3-r28

Shapiro MD, Kronenberg Z, Li C, Domyan ET, Pan H, Campbell M, Tan H, Huff CD, Hu H, Vickrey AI, Nielsen SC, Stringham SA, Hu H, Willerslev E, Gilbert MT, Yandell M, Zhang G, Wang J (2013) Genomic diversity and evolution of the head crest in the rock pigeon. Science 339(6123):1063-1067. doi:10.1126/science. 1230422
Sharman GB (1973) The chromosomes of non-eutherian mammals. In: Chiarelli AN, Capanna E (eds) Cytotaxonomy and vertebrate evolution. Academic, New York, pp 485-530

Shetty S, Griffin DK, Graves JA (1999) Comparative painting reveals strong chromosome homology over 80 million years of bird evolution. Chromosom Res 7(4):289-295

Skinner BM, Griffin DK (2012) Intrachromosomal rearrangements in avian genome evolution: evidence for regions prone to breakpoints. Heredity (Edinb) 108(1):37-41. doi:10.1038/hdy.2011.99

Skinner BM, Robertson LB, Tempest HG, Langley EJ, Ioannou D, Fowler KE, Crooijmans RP, Hall AD, Griffin DK, Volker M (2009) Comparative genomics in chicken and Pekin duck using FISH mapping and microarray analysis. BMC Genomics 10:357. doi:10.1186/1471-2164-10-357

Srikulnath K, Matsubara K, Uno Y, Thongpan A, Suputtitada S, Apisitwanich S, Matsuda Y, Nishida C (2009) Karyological characterization of the butterfly lizard (Leiolepis reevesii rubritaeniata, Agamidae, Squamata) by molecular cytogenetic approach. Cytogenet Genome Res 125(3):213-223. doi:10.1159/ 000230005

Srikulnath K, Uno Y, Nishida C, Matsuda Y (2013) Karyotype evolution in monitor lizards: cross-species chromosome mapping of cDNA reveals highly conserved synteny and gene order in the Toxicofera clade. Chromosome Res. doi:10.1007/s10577-013-9398-0

Stiglec R, Ezaz T, Graves JA (2007) A new look at the evolution of avian sex chromosomes. Cytogenet Genome Res 117(1-4):103-109. doi: $10.1159 / 000103170$

Svartman M, Vianna-Morgante AM (1998) Karyotype evolution of marsupials: from higher to lower diploid numbers. Cytogenet Cell Genet 82(3-4):263-266. doi:10.1159/000015114

Svartman M, Stone G, Page JE, Stanyon R (2004) A chromosome painting test of the basal eutherian karyotype. Chromosom Res 12(1):45-53. doi:10.1023/B:CHRO.0000009294.18760.e4

Thomas JW, Caceres M, Lowman JJ, Morehouse CB, Short ME, Baldwin EL, Maney DL, Martin CL (2008) The chromosomal polymorphism linked to variation in social behavior in the white-throated sparrow (Zonotrichia albicollis) is a complex rearrangement and suppressor of recombination. Genetics 179(3):1455-1468. doi:10.1534/ genetics.108.088229

Trifonov VA, Giovannotti M, O'Brien PC, Wallduck M, Lovell F, Rens W, Parise-Maltempi PP, Caputo V, Ferguson-Smith MA (2011) Chromosomal evolution in Gekkonidae. I. Chromosome painting between Gekko and Hemidactylus species reveals phylogenetic relationships within the group. Chromosome Res 19(7):843-855. doi:10.1007/s10577-011-9241-4

Uno Y, Nishida C, Tarui H, Ishishita S, Takagi C, Nishimura O, Ishijima J, Ota H, Kosaka A, Matsubara K, Murakami Y, Kuratani S, Ueno N, Agata K, Matsuda Y (2012) Inference of the protokaryotypes of amniotes and tetrapods and the evolutionary processes of microchromosomes from comparative gene mapping. PLoS One 7(12):e53027. doi:10.1371/journal.pone.0053027

Valenzuela N, Adams DC (2011) Chromosome number and sex determination coevolve in turtles. Evolution 65(6):1808-1813. doi:10.1111/ j.1558-5646.2011.01258.x

Van Brink JM (1959) Morphological form of digametism in the Sauropsida and the Monotremata. Chromosoma 10(1):1-72

Veyrunes F, Waters PD, Miethke P, Rens W, McMillan D, Alsop AE, Grutzner F, Deakin JE, Whittington CM, Schatzkamer K, Kremitzki CL, Graves T, Ferguson-Smith MA, Warren W, Marshall Graves JA (2008) Bird-like sex chromosomes of platypus imply recent origin of mammal sex chromosomes. Genome Res 18(6):965-973. doi:10. $1101 /$ gr.7101908

Vicoso B, Emerson JJ, Zektser Y, Mahajan S, Bachtrog D (2013) Comparative sex chromosome genomics in snakes: differentiation, evolutionary strata, and lack of global dosage compensation. PLoS Biol 11(8):e1001643. doi:10.1371/journal.pbio.1001643 
Volker M, Backstrom N, Skinner BM, Langley EJ, Bunzey SK, Ellegren H, Griffin DK (2010) Copy number variation, chromosome rearrangement, and their association with recombination during avian evolution. Genome Res 20(4):503-511. doi:10.1101/gr.103663.109

Vonk FJ, Casewell NR, Henkel CV, Heimberg AM, Jansen HJ, McCleary $\mathrm{RJ}$ et al (2013) The king cobra genome reveals dynamic gene evolution and adaptation in the snake venom system. Proc Natl Acad Sci U S A 110(51):20651-20656. doi:10.1073/pnas. 1314702110

Wang Z, Pascual-Anaya J, Zadissa A, Li W, Niimura Y, Huang Z et al (2013) The draft genomes of soft-shell turtle and green sea turtle yield insights into the development and evolution of the turtlespecific body plan. Nat Genet 45(6):701-706. doi:10.1038/ng.2615

Warren WC, Hillier LW, Marshall Graves JA, Birney E, Ponting CP, Grutzner F et al (2008) Genome analysis of the platypus reveals unique signatures of evolution. Nature 453(7192):175-183. doi:10. 1038/nature06936

Warren WC, Clayton DF, Ellegren H, Arnold AP, Hillier LW, Kunstner A et al (2010) The genome of a songbird. Nature 464(7289):757-762. doi:10.1038/nature08819

Westerman M, Meredith RW, Springer MS (2010) Cytogenetics meets phylogenetics: a review of karyotype evolution in diprotodontian marsupials. J Hered 101(6):690-702. doi:10.1093/jhered/esq076
Wrigley JM, Graves JA (1988a) Karyotypic conservation in the mammalian order monotremata (subclass Prototheria). Chromosoma 96(3): 231-247

Wrigley JM, Graves JA (1988b) Sex chromosome homology and incomplete, tissue-specific $\mathrm{X}$-inactivation suggest that monotremes represent an intermediate stage of mammalian sex chromosome evolution. J Hered 79(2):115-118

Yang F, O’Brien PC, Milne BS, Graphodatsky AS, Solanky N, Trifonov V, Rens W, Sargan D, Ferguson-Smith MA (1999) A complete comparative chromosome map for the dog, red fox, and human and its integration with canine genetic maps. Genomics 62(2): 189-202. doi:10.1006/geno.1999.5989

Young GJ, Graves JAM, Barbieri I, Woolley PA, Cooper DW, Westerman M (1982) The chromosomes of dasyurids (Masupialia). In: Archer M (ed) Carnivorous Marsupials vol 2. Royal Zool. Soc., NSW, pp 783-795

Young MJ, O’Meally D, Sarre SD, Georges A, Ezaz T (2013) Molecular cytogenetic map of the central bearded dragon, Pogona vitticeps (Squamata: Agamidae). Chromosom Res 21(4):361-374. doi:10. 1007/s10577-013-9362-z

Zhan X, Pan S, Wang J, Dixon A, He J, Muller MG et al (2013) Peregrine and saker falcon genome sequences provide insights into evolution of a predatory lifestyle. Nat Genet 45(5):563-566. doi:10.1038/ng. 2588 